\title{
EVALUATION OF A GROUP OF PYRROLE DERIVATIVES AS TUBERCULOSTATIC AGENTS
}

\author{
Maya Georgieva, ${ }^{1}$ Diana Tzankova, ${ }^{2}$ Stanislava Vladimirova, ${ }^{3}$ Atanas Bijev ${ }^{4}$
}

\begin{abstract}
This research aims to contribute to the global search for more effective tuberculostatics that has been triggered by recent outbreaks of tuberculosis. A group of pyrrole-containing derivatives are designed and theoretically elucidated. The identification of the pharmacophore group using the PharmaGist webserver is attempted. Also, the corresponding drug-like properties of the tested compounds are evaluated, together with their possible toxicity risks. The pharmacokinetic behavior of the structures is predicted, based on the Lipinski's Rule of Five. The effects of some structural parameters are tested. In addition, in vitro evaluations of the anti-tubercular activity against Mycobacterium tuberculosis $\mathrm{H}_{37} \mathrm{Rv}$ are performed, with compound GA-9 registering the highest activity.
\end{abstract}

UDC Classification: 615.3; DOI: http://dx.doi.org/10.12955/cbup.v5.1075

Keywords: pyrrole-derivatives, pharmacophore, drug-likeness, tuberculostatic

\section{Introduction}

According to recent statistics published by the World Health Organization (WHO), tuberculosis (TB) is one of the top 10 causes of death worldwide. In 2015, 10.4 million people were infected with TB, with 1.8 million deaths, including 0.4 million among them with the human immunodeficiency virus (HIV). Over $95 \%$ of TB deaths occur in low- and middle-income countries, where, according to WHO (2017):

Six countries account for $60 \%$ of the total deaths, with India leading the count, followed by Indonesia, China, Nigeria, Pakistan and South Africa. In 2015, an estimated 1 million children were infected with TB and 170000 children died of TB (excluding children with HIV). TB is a leading killer of HIV-positive people: in 2015, 35\% of HIV deaths were due to TB. Globally in 2015, an estimated 480000 people developed multidrug-resistant TB (MDR-TB) (APA: WHO Tuberculosis, 2017).

Considering these statistics, "ending the TB epidemic by 2030 is among the health targets of the newly adopted by WHO Sustainable Development Goals" (WHO Tuberculosis, 2017).

Also, according to Euro WHO (n.d.):

Bulgaria is among the 18 high priority countries for tuberculosis (TB) control in the WHO European Region. Challenges for the National Tuberculosis Programme (NTP) include the lack of efficient TB infection control and the low level of engagement of TB care providers, especially primary health care providers, in early detection and follow-up of TB patients (APA: Euro WHO, 2017).

Thus, in the search for a promising platform of structures for evaluating anti-tubercular agents, a significant number of investigations have focused on a variety of heterocyclic derivatives, including structures containing a pyrrole heterocycle, such as the known anti-tubercular agent BM 212 (Deidda, 1998) and its analogs (Poce, 2013).

In the same context, a series of substituted pyrroles were synthesized at the Faculty of Pharmacy, Medical University -Sofia, Bulgaria and laboratory of organic synthesis at University of Chemical Technology and Metallurgy (UCTM) - Bulgaria and evaluated as anti-tubercular agents, with up to $100 \%$ inhibitory activity against Mycobacterium tuberculosis or a half maximal inhibitory concentration (IC50) less than $6 \mu \mathrm{g} \mathrm{ml}^{-1}$ (Bijev, 2010; Georgieva, 2010; Georgieva, 2011). In addition, reliable structure-activity relationships have been derived (Lessigiarska, 2012) that attempt to contribute to the global fight against the spread of tuberculosis.

\footnotetext{
${ }^{1}$ Medical University, Faculty of Pharmacy, Sofia, Bulgaria, georgm@mail.bg

${ }^{2}$ Medical University, Faculty of Pharmacy, Sofia, Bulgaria, diana_ducova@abv.bg

${ }^{3}$ University of Chemical Technology and Metallurgy, Sofia, Bulgaria, vladimirova.s@mail.bg

${ }^{4}$ University of Chemical Technology and Metallurgy, Sofia, Bulgaria, atanas.bijev@gmail.com
} 


\section{Design and Synthesis of the Evaluated Structures}

A variety of chemical templates has been explored by us (Bijev, 2006), including hydrazones (Bijev, 2008a) and derivatives of pyrrole (Bijev, 2008b). The performed analysis motivated us to combine the active principles of these moieties, and thus we synthesized consecutively several series of such pyrrole derivatives as potential anti-TB (anti-tubercular) agents (Bijev, 2006; Bijev,2008a; Bijev, 2008b; Bijev, 2010; Georgieva, 2010; Vladimirova, 2015).

This research aims to contribute towards the global search for more effective tuberculostatics. The design of the molecules included in this study is addressed to diversify the available pyrrole derivatives already identified as perspective inhibitors of M. tuberculosis.

\section{Data and Methodology}

This study examined structural changes in three focus areas identified as A, B, and C of targeted compounds (Figure 1).

Figure 1: Specific moieties in the targeted compounds

This approach was based on observations of Vladimirova \& Bijev, (2015, p. 5): "The most radical change was made in moiety A, whereat the $\mathrm{N}$-acyl-chain in the previously developed pyrroles in our laboratory was replaced by $\mathrm{R}_{1}-\mathrm{R}_{3}$-substituted phenyl", which was "motivated by the presence of the same moiety in series of bioactive products."

In an attempt to evaluate the independent effect of this new moiety $A$, the ester group $\left(\mathrm{R}=\mathrm{OC}_{2} \mathrm{H}_{5}\right)$ in moiety $\mathrm{B}$ was maintained in three of the products. Aiming for an analogy with the piperazine- or thiomorpholine-heterocycle in BM 212 and their analogs, a change in moiety $\mathrm{B}$ with $\mathrm{R}=\mathrm{CH}_{3}$ or a morpholino-substituent was expected. In group fragment $\mathrm{C}, \mathrm{X}=\mathrm{H}$ alternates with $\mathrm{X}=\mathrm{Cl}$ aimed to identify some similarity to substituted active tuberculostatics previously developed by the authors (Georgieva, 2010; Biava, 2004; Biava, 2008).

The products targeted in this study as described elsewhere by Vladimirova (2015, p. 3): "were synthesized via adopted Paal-Knorr cyclization by condensation of three 1,4-dicarbonyl compounds with a set of substituted anilines."

\section{Identification of the Pharmacophores}

According to Schneidman-Duhovny, Dror, Inbar, Nussinov, and Wolfson (2008, p. 1), "Predicting molecular interactions is a major goal in rational drug design." They also stated that: "The spatial arrangement of features, identified as pharmacophore group and responsible for the interaction of the designed molecule with a desired target receptor, is an important model for achieving" this purpose (Schneidman-Duhovny, Dror, Inbar, Nussinov, and Wolfson (2008, p. 1)).

This study aimed to identify the pharmacophore group using the PharmaGist Webserver (SchneidmanDuhovny, Dror, Inbar, Nussinov, and Wolfson, 2008). The evaluated compounds were separated into two groups, according to their structural similarity to the published pyrrole agent with underlined antituberculosis activity, BM 212 (Biava, 2004; Figure 2). 


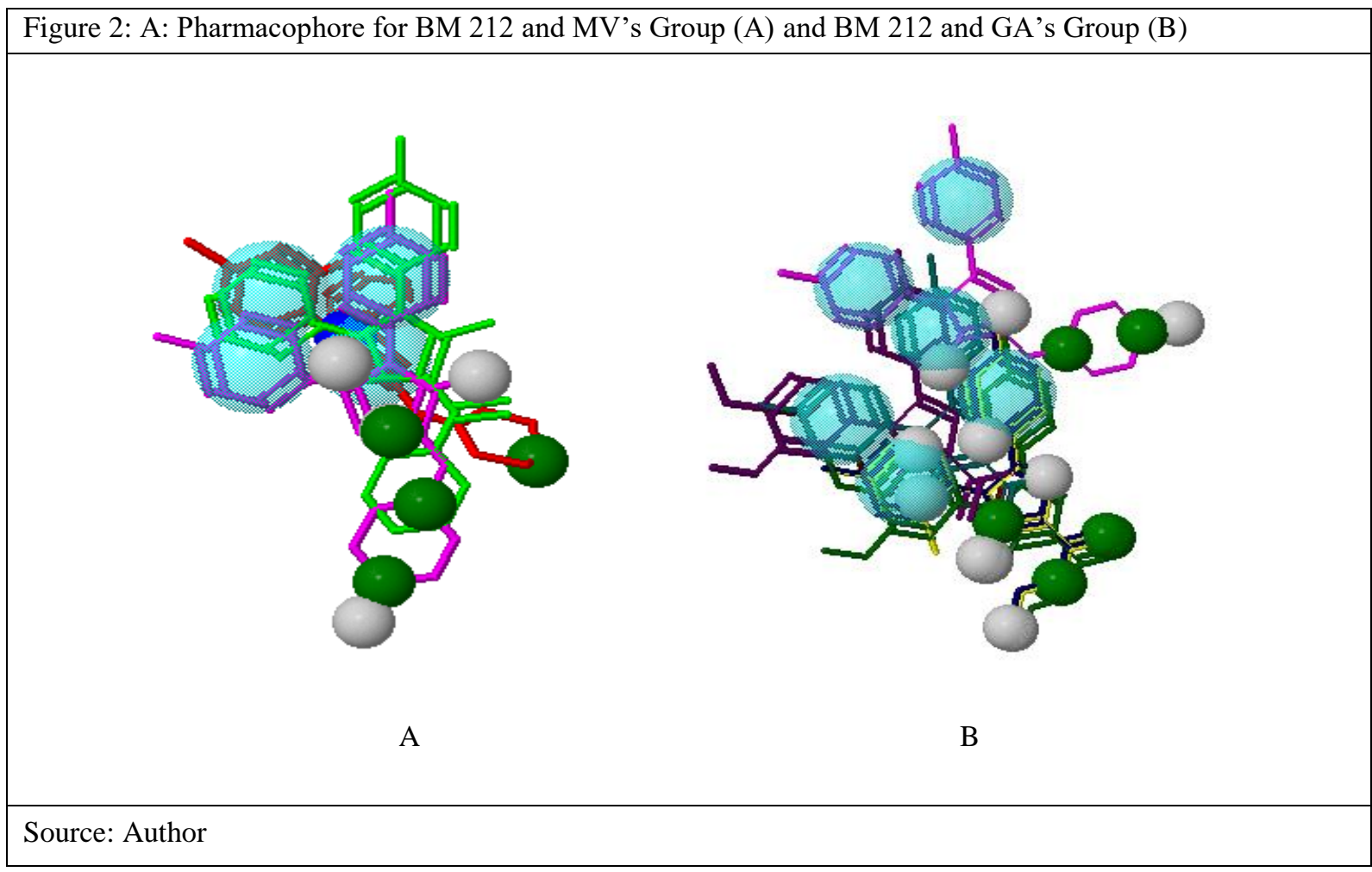

A ligand based method was employed, and the candidate pharmacophores were outlined, computed by multiple flexible alignments of the input ligands. The method explicitly considers the flexibility of the input ligands within the alignment process (Schneidman-Duhovny, Dror, Inbar, Nussinov, and Wolfson, 2008).

\section{Drug-likeness Prediction}

There are many approaches that assess a compound's 'drug-like' properties, partially based on topological descriptors, fingerprints of MDL structure keys or other properties.

The biological activity is also identified as a function of the complex influence of a variety of molecular descriptors. The underlayment of a certain structural parameter and the expected pharmacological effect is at times possible. The hydrophobicity of the compounds expressed as $\log \mathrm{P}$ outlined the key parameter for determination of compounds Drug-likeness.

The study aimed to predict the drug-like properties of the newly synthesized pyrrole derivatives using the following two approaches: Lipinski's Rule of Five limitations (Lipinski, 1997; Lipinski, 2001) and OSIRIS Property Explorer evaluation score (Sander, Thomas Actelion Pharmaceuticals Ltd., 2017). The OSIRIS Property Explorer prediction tool used in this publication is an integral part of Actelio's in-house substance registration system (Sander, Thomas Actelion Pharmaceuticals Ltd., 2017). The prediction process relied on a precomputed set of structural fragments that provided preliminary information on a number of drug-relevant properties, such as drug-likeness and toxicity alerts (Sander, Thomas Actelion Pharmaceuticals Ltd., 2017).

\section{Prediction of the Pharmacokinetic Behavior}

The pharmacokinetic of the evaluated structures was important for initial information in their identification as possible active agents. The behavior of the targeted structures was easily predicted, based on the results from the performed elucidation of Lipinski's Rule of Five parameters pointing the following boundaries: hydrophobic parameter $\log \mathrm{P}<5$, molecular weight $\mathrm{MW}<500$, the number of hydrogen bond acceptors $\mathrm{n}(\mathrm{O}, \mathrm{N})<10$, and the number of hydrogen bond donors $\mathrm{n}(\mathrm{OH}, \mathrm{NH})<5$ (Lipinski, 1997; Lipinski, 2001). The desired parameters were calculated with the help of online cheminformatics services offered by Molinspiration Cheminformatics.

\section{Evaluation of Drug-likeness}

The synthesized new derivatives of pyrrole were assessed for drug-likeness using two parameters: Fragment Based Drug-likeness and Drug Score. 
The Fragment Based Drug-likeness was calculated by the following equation for summing score values of those fragments that were present in the molecule under investigation, as follows:

$$
d=\frac{\sum v_{i}}{\sqrt{n}}
$$

A positive value meant that the analyzed molecule contained predominantly fragments that are frequently in commercial drugs (Sander, 2017).

The Drug Score was the combined value of the drug-likeness, cLogP, $\log S$, molecular weight, and toxicity risks, used to evaluate the compound's overall potential to qualify as a drug. It was calculated by multiplying contributions of the individual properties with Equation 1:

$$
d s=\prod\left(\frac{1}{2}+\frac{1}{2} S_{i}\right) \cdot \Pi t_{i}
$$

where $d s$ is the drug score and $\mathrm{S}_{i}$ the contributions calculated directly from of cLogP, $\log$, mol weight and drug-likeness $\left(\mathrm{p}_{i}\right)$ using Equation 3, which describes a spline curve:

$$
S_{i}=\frac{1}{1+e^{a p_{i}+b}}
$$

where, respectively, $a$ and $b$ were the values of $\operatorname{cLogP}(1,-5), \operatorname{logS}(1,5)$, mol weight $(0.012,-6.000)$, and drug-likeness $(1,0)$. The $t_{i}$ was the contribution obtained for the four toxicity risk types. The $t_{i}$ values were 1.0, 0.8 and 0.6 for 'no risk', 'medium risk', and 'high risk', respectively (Sander, 2017).

\section{Screening for Tuberculostatic Activity}

The anti-tubercular activities of the target compounds were determined by in vitro procedure at the SRI International, a contractor for National Institute of Allergy and Infectious Diseases (NIAID), USA (SRI International, 2010).

The evaluation was performed according to the SRI International Screening Program. This involved a single point concentration procedure where all compounds were initially screened against $M$. tuberculosis strain $\mathrm{H}_{37} \mathrm{Rv}$ (Collins, 1997). This assay was the primary screen. Where compounds were active at $10 \mu \mathrm{g} \mathrm{mL}^{-1}$ level, they were further tested in a minimal inhibitory concentration (MIC) assay at eight concentrations in a dose range between 10.000 and $0.078 \mu \mathrm{g} \mathrm{mL}^{-1}$ (Georgieva, 2010).

The procedure was performed as follows: $20 \mu \mathrm{l}$ of the $3.2 \mathrm{mg} \mathrm{mL}^{-1}$ test compound was added to a 96well microtiter plate. Two-fold dilutions were made by adding $20 \mu \mathrm{l}$ of diluent. Each dilution was further diluted 1:10 in sterile water ( $10 \mu \mathrm{l}$ of dilution to $90 \mu \mathrm{l}$ of sterile water). An amount of $6.25 \mu \mathrm{l}$ of each dilution was transferred to duplicate 96 -well test plates. Then, $93.75 \mu 1$ of the cell suspension ( 10 $10^{4}$ bacteria) in $7 \mathrm{H} 9$ medium was added to the test plates. Positive (included rifampicin and isoniazid), negative (included cell culture with solvent and water, and cell culture only), sterility (included media only; and media with solvent and water) and Resazurin (7-Hydroxy-3H-phenoxazin3-one 10-oxide; included one plate containing the diluted compound with Resazurin only) controls were tested. The 96-well test plates were incubated at ambient $37^{\circ} \mathrm{C}$ incubator for six days. After the 6-day incubation, $5 \mu \mathrm{l}$ of a $0.05 \%$ sterile Resazurin solution was added to each well of the 96-well plate. The plates were placed in an ambient $37^{\circ} \mathrm{C}$ incubator for two days. After the 2-day incubation, a visual evaluation and a fluorimetric reading were performed. The results were presented as ' $\%$ Growth Inhibition' (Georgieva, 2010).

The tuberculostatic activity of the tested series of pyrrole derivatives against $M$. tuberculosis, strain $\mathrm{H}_{37} \mathrm{Rv}$, evaluated by SRI International as '\% Growth Inhibition' was transformed into a more common general format in an attempt to identify possible trends. Thus, with the help of simplifying considerations, the current results were recalculated from ' $\%$ Growth Inhibition' to '\% inhibitory activity' related to that of BM 212 (accepted to be a standard within 100\%). The following approximations were applied:

Recalculated $\%$ inhibitory activity $=\%$ Growth Inhibition +7.13

where the value of 7.13 was the absolute poorest registered (with negative sign) activity (Georgieva, 2010).

\section{Results and Discussion}

The best pairwise solution for the evaluated molecules and BM 212 was its pairing with MV-3 (Figure $3)$. 


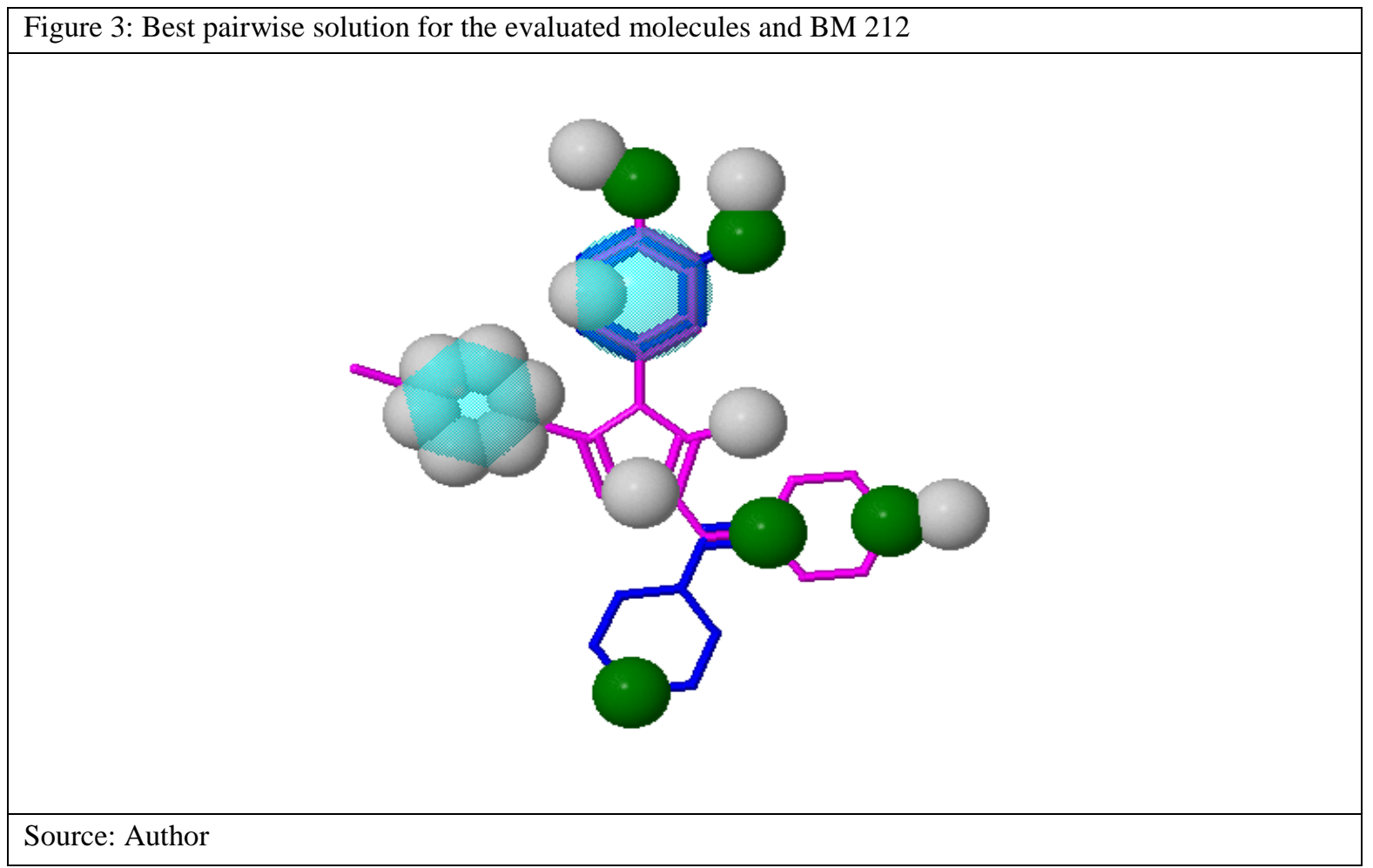

The identified pharmacophores for the most active agent identified from the microbiological testing and then used as reference agent BM 212 are shown in Figure 4.

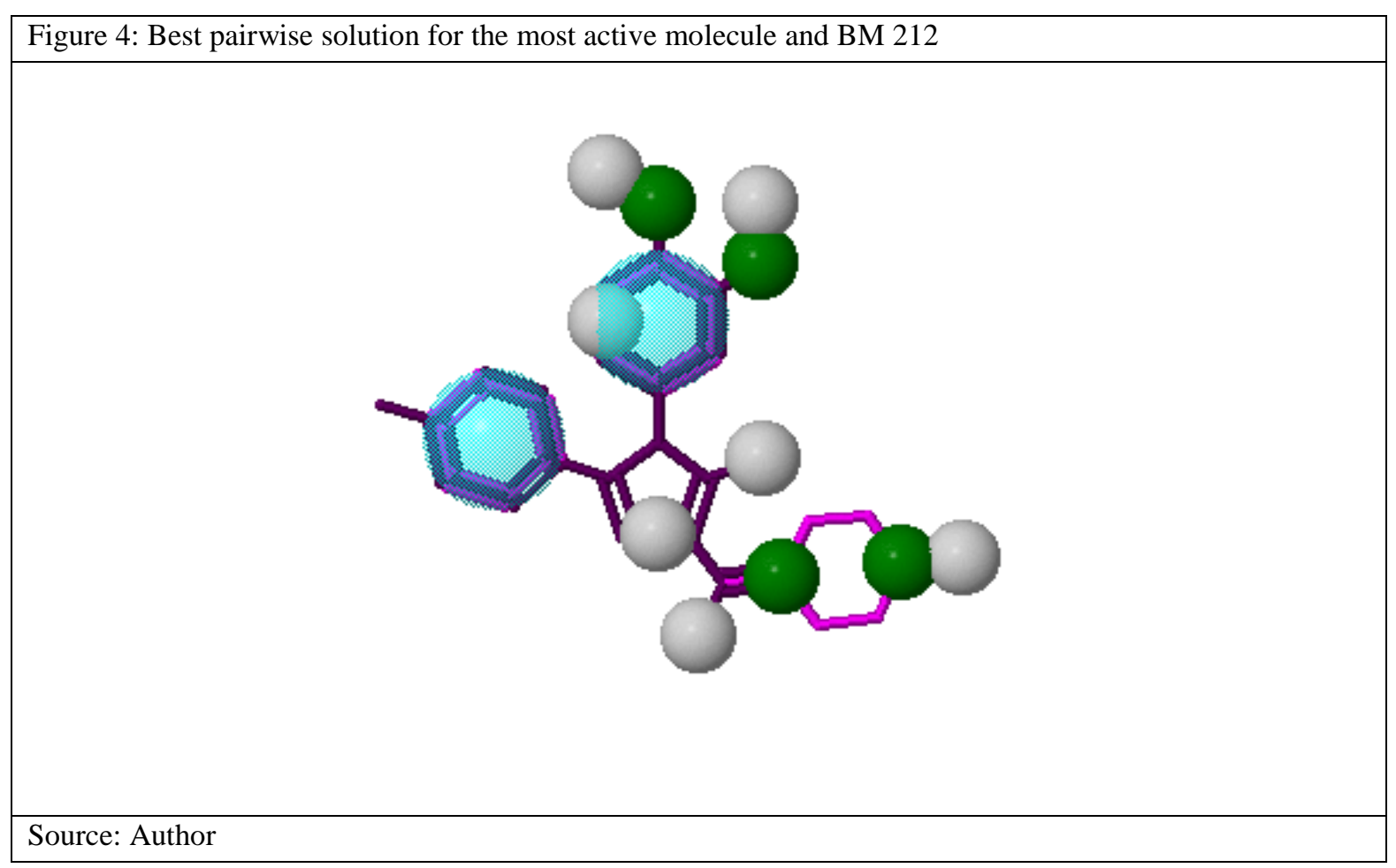

The identified pharmacophores may be further used for generating new leads and optimizing existing ones or both in designing drugs.

The results of the performed calculations for prediction of the pharmacokinetic behavior are presented in Table 1. 


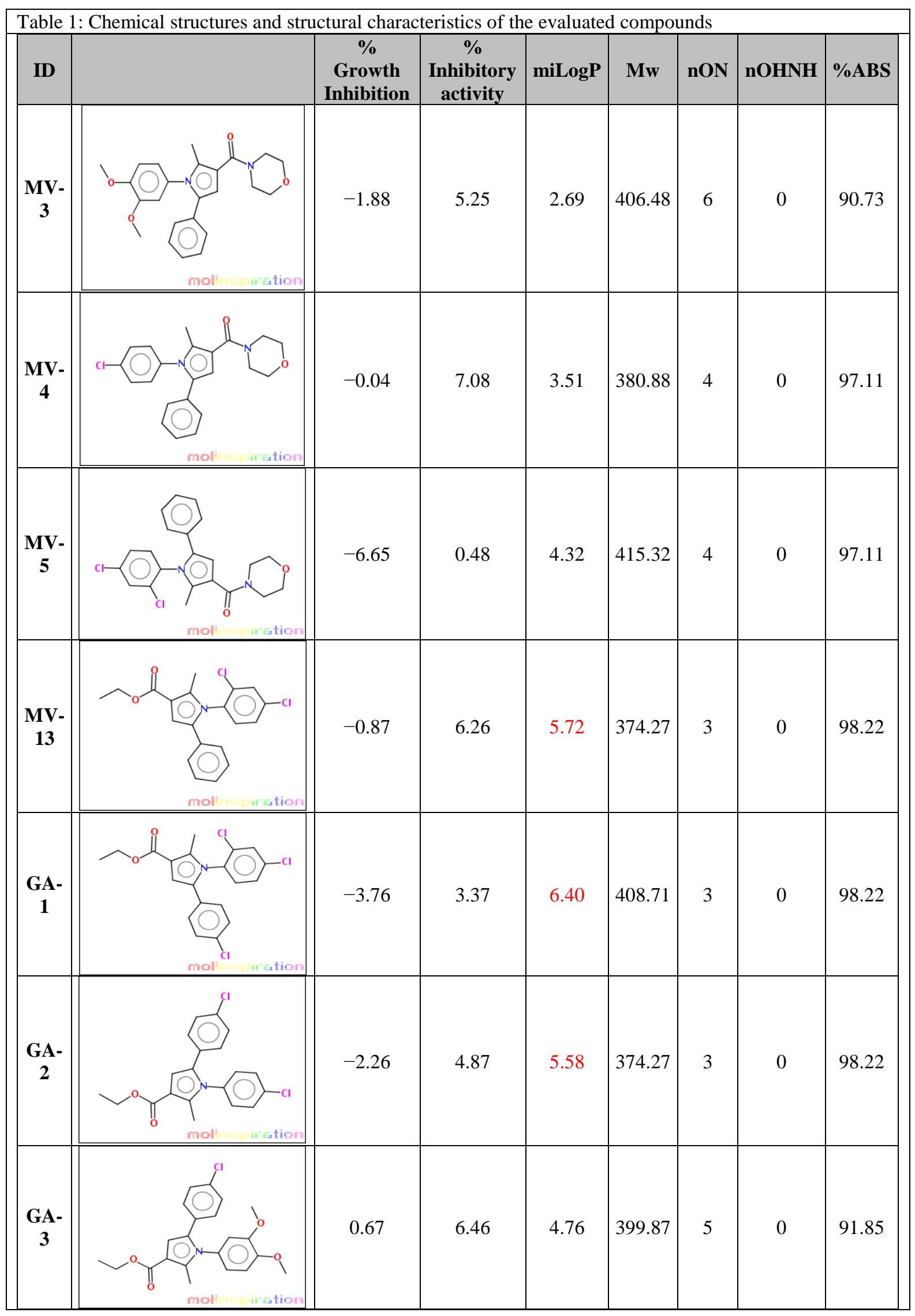




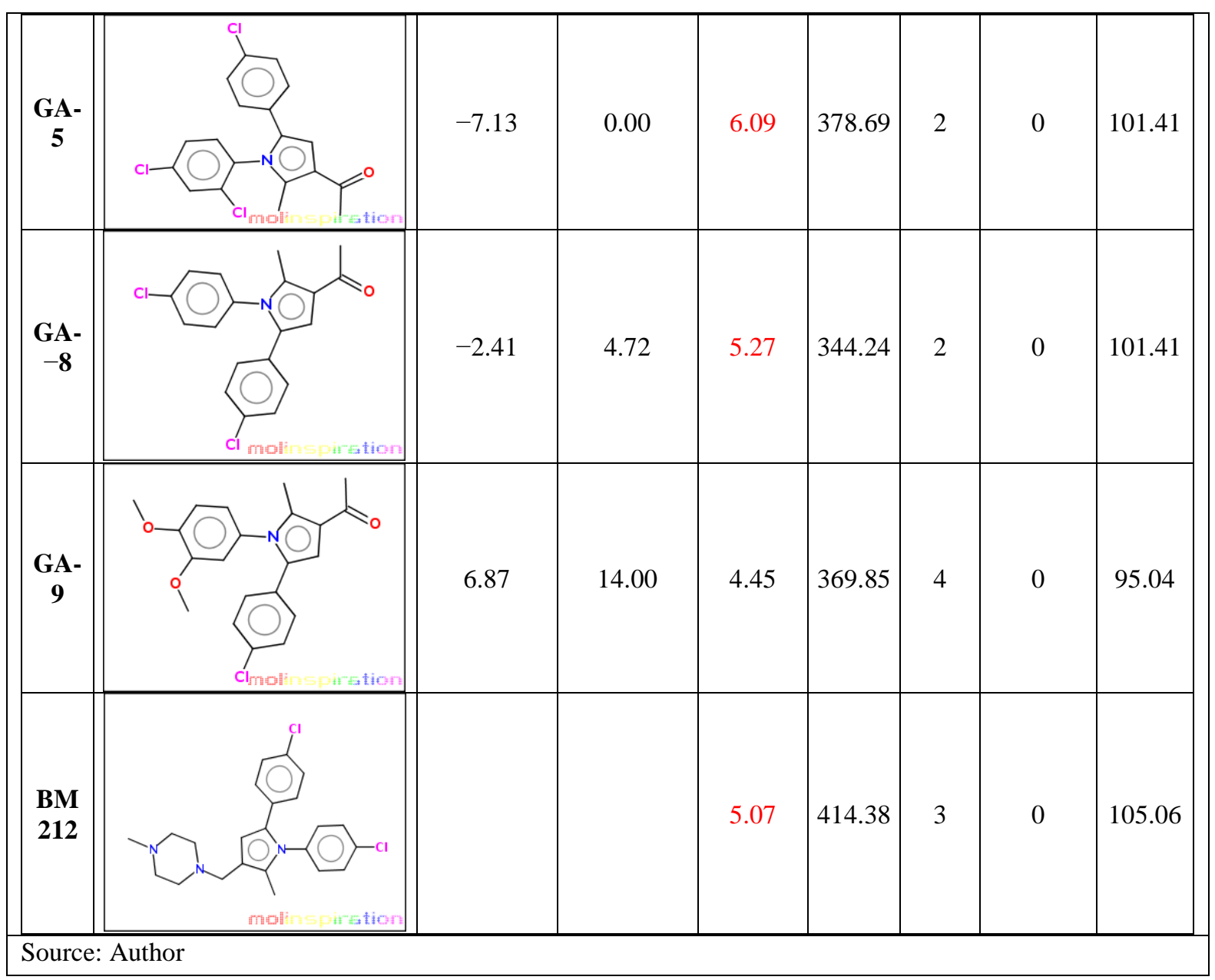

The compounds MV-13, GA-1, GA-2, GA-5, and GA-8 violated the rule with the miLogP parameter. As the structures are considered antimicrobials, the observed violations do not affect their value as pharmacokinetics.

Another confirmation was the result from the performed prediction with the absolute value (\%ABS), calculated by the expression: \%ABS $=109-0.345$ TPSA introduced elsewhere (Zhao, 2002).

The results showed the target compounds had commensurable and high \%ABS, which indicates valuable pharmacokinetics and thus indicated they follow the regulations of Lipinski's Rule of Five, decreasing any pharmacokinetic concerns.

The corresponding values of the computational analysis for Drug-likeness and Drug Score are presented in Table 2 .

\begin{tabular}{|c|c|c|c|c|c|c|}
\hline ID & Mutagenic & Tumorigenic & Irritant & Reproductive effective & Drug-likeness & Drug-score \\
\hline MV-3 & $\checkmark$ & - & - & - & 0.52 & 0.34 \\
\hline MV-4 & - & - & - & - & 3.44 & 0.43 \\
\hline MV-5 & - & - & - & - & 3.67 & 0.35 \\
\hline MV-13 & - & - & - & - & 4.60 & 0.49 \\
\hline GA-1 & - & - & - & - & -2.14 & $\underline{0.15}$ \\
\hline GA-2 & - & - & - & - & -2.96 & $\underline{0.17}$ \\
\hline GA-3 & $\checkmark$ & - & - & - & $\overline{-5.12}$ & $\overline{0.15}$ \\
\hline GA-5 & - & - & - & - & $\overline{1.63}$ & $\overline{0.27}$ \\
\hline GA-8 & - & - & - & - & 0.79 & 0.29 \\
\hline GA-9 & $\checkmark$ & - & - & - & -1.46 & $\underline{0.19}$ \\
\hline BM 212 & - & - & - & - & $\overline{4.60}$ & $\overline{0.49}$ \\
\hline
\end{tabular}

Source: Author 
The evaluation established that compound MV-13 shows the highest drug like properties with Fragment Based Drug-likeness score of 4.60 and total Drug Score of 0.49, equal to the new antitubercular agent BM 212.

The primary screening results of the evaluated compounds are presented in Figure 5.

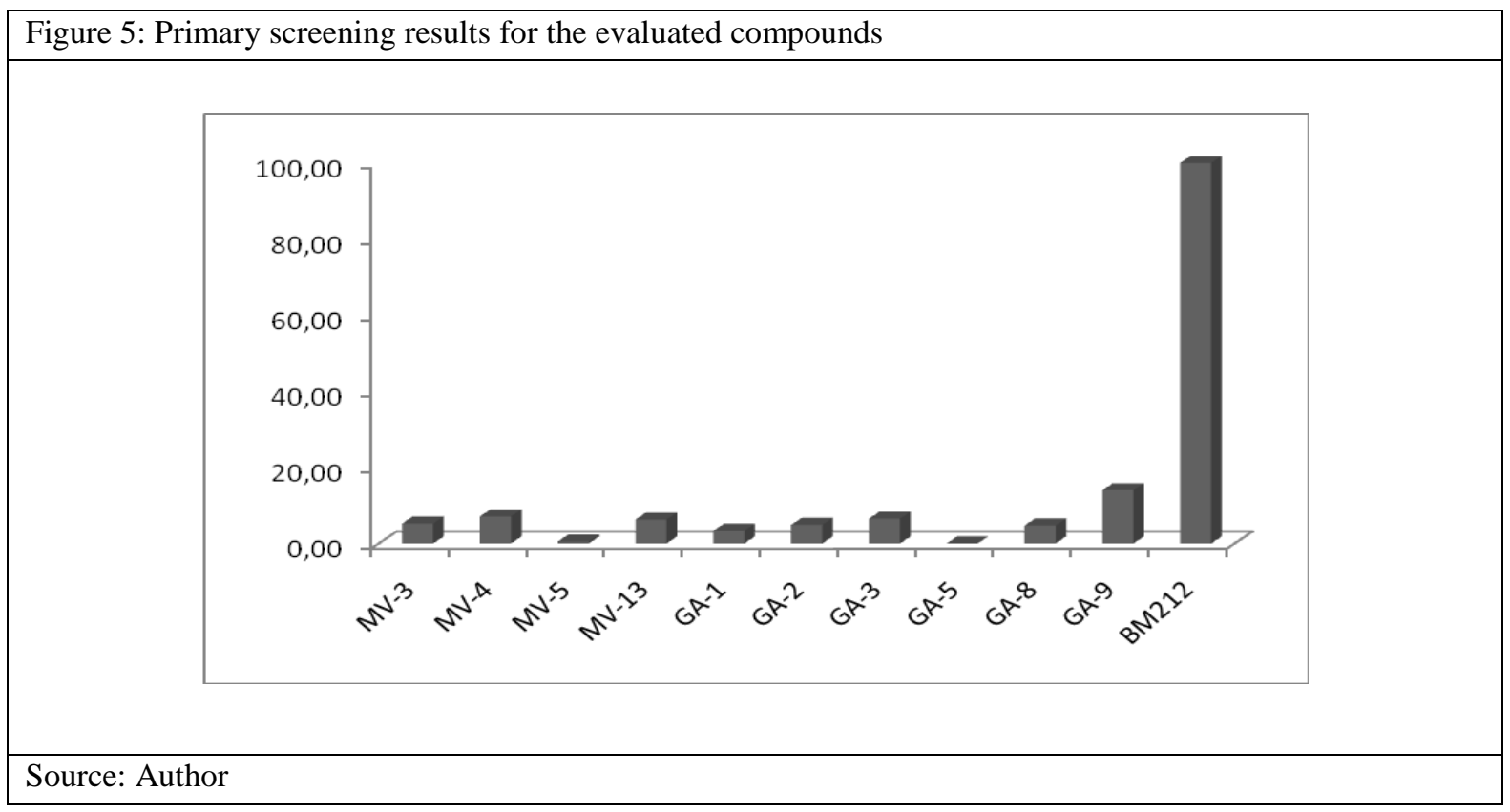

From the test group, compound GA-9 showed the highest inhibitory activity. This result confirms the expectation of the drug-like effect of the underlined structure, based on the drug-like similarity with the new anti-tubercular agent BM 212.

\section{Conclusion}

The results indicate that the identified pharmacophores have additional use in generating new leads or optimizing existing ones or both in drug design. The newly synthesized products strongly aligned with Lipinski's Rule of Five limitations, which is a premise for sound pharmacokinetics. In addition, the target compounds had commensurable and high absolute values. On the one hand, a comparison of results showed five compounds failed to meet Lipinski's Rule of Five parameters, with these compounds unsuitable for further structural optimizations. On the other hand, the compound MV-13 had the highest drug-like properties, with a Fragment Based Drug-likeness score of 4.60 and total Drug score of 0.49, equal to the new anti-tubercular agent BM 212. As well, compound GA-9 was underlined as having the highest tuberculostatic activity amongst the evaluated molecules.

\section{References}

APA: Who | Tuberculosis. (2017.). Retrieved from http://www.who.int/mediacentre/factsheets/fs104/en/?ncid=newsltushpmg00000003)

APA: Who/europe | Bulgaria. (n.d.). Retrieved from http://www.euro.who.int/en/health-topics/communicablediseases/tuberculosis/coun

Biava, M., Porretta, G. C., Poce, G., De Logu, A., Saddi, M., Meleddu, R., Manetti, F., De Rossi, E., \& Botta M. (2008). 1,5Diphenylpyrrole Derivatives as Antimycobacterial Agents. Probing the Influence on Antimycobacterial Activity of Lipophilic Substituents at the Phenyl Rings. J. Med. Chem., 51 (12), 3644-3648.

Biava, M., Porretta, G., Deidda, D., Pompei, R., \& Tafi, A. (2004). Antimycobacterial compounds. New pyrrole derivatives of BM212. Bioorganic \& Medicinal Chemistry, 12, 1453-1458.

Bijev, A. (2006). New hydrazones as pyrrole derivatives with higher inhibitory activity against Mycobacterium tuberculosis. Lett Drug Des Discov, 3(7), 506-512.

Bijev, A. (2008a). Synthesis, in vitro evaluations and structure-activity assessment of pyrrole hydrazones. Lett Drug Des Discov, 5(1), 15-24.

Bijev, A., \& Georgieva, M. (2010). Pyrrole-Based Hydrazones Synthesized and Evaluated In Vitro as Potential Tuberculostatics. Letters in Drug Design \& Discovery, 7 (6), 430-437.

Bijev, A. (2008b). Synthesis and in vitro Evaluation of New Hydrazones as Pyrrole Derivatives with Anti-tubercular Activity. Arzneim-Forsch/Drug Res. 
Collins, L., \& Franzblau S. G. (1997). Microplate Alamar Blue Assay versus BACTEC 460 system for high-throughput screening of compounds against Mycobacterium tuberculosis and Mycobacterium avium. Antimicrob Agents Chemother, 41, 1004-1009.

Deidda, D., Lampis, G., Fioravanti, R., Biava, M., \& Porretta, G.C. (1998). Bactericidal activities of the pyrrole derivative BM 212 against multidrug-resistant and intramacro-phagic Mycobacterium tuberculosiss trains. Antimicrob Agents Chemother, 42, 3035-3037.

Georgieva, M., Bijev, A., \& Nencheva, I. (2011). Isolation and characterization of isomers of pyrrole-hydrazones with possible tuberculostatic activity. Comparison of methods for separation. Pharmacia, LVIII, 26-31.

Georgieva, M., Bijev, A., \& Prodanova, P. (2010). Synthesis and comparative study of tuberculostatic activity of pyrrolebased hydrazones related to structural variations. Pharmacia, 57 (1-4), 3-14.

http://www.euro.who.int/en/health-topics/communicable-diseases/tuberculosis/country-work/bulgaria/, Accessed on 15 January 2017.

http://www.who.int/mediacentre/factsheets/fs104/en/, Accessed on 15 January 2017.

Lessigiarska, I., Pajeva, I., Prodanova, P., Georgieva, M., \& Bijev, A. (2012). Structure-activity relationships of pyrrole hydrazones as new anti-tuberculosis agents. Medicinal Chemistry Med Chem, 8 (3), 462-473.

Lipinski, C. A., Lombardo, F., Dominy, B.W., \& Feeney, P. (1997). Experimental and computational approaches to estimate solubility and permeability in drug discovery and development settings. J. Adv. Drug Deliv. Rev., 23, 3.

Lipinski, C. A., Lombardo, F., Dominy, B.W., \& Feeney, P.J. (2001). Experimental and computational approaches to estimate solubility and permeability in drug discovery and development settings. Adv Drug Delivery Rev, 46, 3-26.

Molinspiration Cheminformatics, http://www.molinspiration.com/ (accessed on Jan17th, 2016).

Petrović, V. P., Simijonović, D., Živanović, M. N., Košarić, J. V., Petrović, Z. D., Marković, S., \& Marković, S. D. (2014). Vanillic Mannich bases: synthesis and screening of biological activity. Mechanistic insight into the reaction with 4chloroaniline. RSC Adv., 4, 24635-24644.

Poce, G., Bates, R. H., Alfonso, S., Cocozza, M., Porretta, G. C., Ballell, L., Rullas, J., Ortega, F., De Logu, A., Agus, E., La Rosa, V., Pasca, M. R., De Rossi, E., Wae, B., Franzblau, S. G., Manetti, F., Botta, M. \& Biava M. (2013). Improved BM212 MmpL3 Inhibitor Analogue Shows Efficacy in Acute Murine Model of Tuberculosis Infection, PLoS One, 8 (2), e56980.

Sander, Thomas Actelion Pharmaceuticals Ltd., Gewerbestrasse 16, 4123 Allschwil, Switzerland, Retrieved January, 16, 2017 from http://www.organic-chemistry.org/prog/peo/.

Sander, Thomas Actelion Pharmaceuticals Ltd., Gewerbestrasse 16, 4123 Allschwil, Switzerland, Retrieved January, 16, 2017 from http://www.organic-chemistry.org/prog/peo/Drug-likeness.html.

Sander, Thomas Actelion Pharmaceuticals Ltd., Gewerbestrasse 16, 4123 Allschwil, Switzerland, Retrieved January, 16, 2017 from http://www.organic-chemistry.org/prog/peo/drugScore.html.

Schneidman-Duhovny, D., Dror, O., Inbar, Y., Nussinov, R., \& Wolfson H. J. (2008). PharmaGist: a webserver for ligandbased pharmacophore detection. Nucleic Acids. Research, 1-6

SRI International Screening Program on line: http://www.niaid.nih.gov/LabsAnd

Resources/resources/dmid/pretheraagents/Pages/preclinexamples.aspx (accessed on 26 January 2010).

SRI International, Home page: http://www.sri.com (accessed on 26 January 2010).

Vladimirova, S., \& Bijev, A. (2015). Synthesis of new derivatives of pyrrole tuberculostatics providing structural diversity. Pharmacia, 62(1), 3-8.

Zhao, Y. H., Abraham, M. H., Lee, J., Hersey, A., Luscombe, Ch. N., Beck, G., Sherborne, B., \& Cooper, I. (2002) Ratelimited steps of human oral absorption and QSAR studies. Pharm. Res., 19, 1446-1457. 